\title{
Increased Nitric Oxide Synthase Activity Despite Lack of Response to Endothelium-dependent Vasodilators in Postischemic Acute Renal Failure in Rats
}

\author{
John Conger, John Robinette, Adolfo Villar, Leopoldo Raij, " and Pamela Shultz* \\ Department of Medicine, University of Colorado Health Sciences and Veterans Administration Medical Centers, Denver, Colorado \\ 80220; and *Department of Medicine, Veterans Administration Medical Center, and University of Minnesota, Minneapolis, \\ Minnesota 55417
}

\begin{abstract}
Lack of response to endothelium-dependent vasodilators generally has been considered to be evidence for decreased nitric oxide synthase (NOS) activity and NO generation after ischemic or hypoxic injury to vital organs including the kidney. In this study, renal blood flow (RBF) responses to endothelium-dependent vasodilators acetylcholine and bradykinin and the endothelium-independent vasodilator prostacyclin, the nonselective NOS inhibitor L-NAME (without and with L-arginine), the inducible NOS inhibitor aminoguanidine, and the NO-donor sodium nitroprusside were examined in 1-wk norepinephrine-induced (NE) and sham-induced acute renal failure (ARF) rats. Compared with sham-ARF, there was no increase in RBF to intrarenal acetylcholine and bradykinin, but a comparable RBF increase to prostacyclin in NE-ARF kidneys. However, there was a significantly greater decline in $R B F$ to intravenous $L$ NAME in NE- than sham-ARF rats $(-65 \pm 8$ vs. $-37 \pm 5 \%, P$ $<0.001$ ) which was completely blocked by prior $L$-arginine infusion. There was no change in RBF to the inducible NOS specific inhibitor aminoguanidine. Unlike sham-ARF, there was no increase in RBF to intrarenal sodium nitroprusside in NE-ARF. Immunohistochemistry and immunofluorescence detection of constitutive (c) NOS using mouse monoclonal antibody were carried out to positively determine the presence of cNOS in NE-ARF. $90 \%$ of renal resistance vessels showed evidence of endothelial cNOS in both sham- and NE-ARF. Taken together, results of these experiments are consistent with the conclusion that NOS/NO activity is, in fact, maximal at baseline in 1-wk NE-ARF and cannot be increased further by exogenous stimuli of NOS activity. The increased NOS is likely of the constitutive form and of endothelial origin. It is suggested that the increased NOS activity
\end{abstract}

This work was presented in part at the Annual Meeting of the American Society of Nephrology in Boston on 14 November 1993 and published in abstract form (1993. J. Am. Soc. Nephrol. 4:743A).

Address correspondence to John D. Conger, M.D., VA Medical Center (151), 1055 Clermont Street, Denver, CO 80220. Phone: 303393-2892; FAX: 303-377-5686.

Received for publication 30 June 1994 and accepted in revised form 6 April 1995.

The Journal of Clinical Investigation, Inc.

Volume 96, July 1995, 631-638 is in response to ischemia-induced renal vasoconstrictor activity. Attenuated response to endothelium-dependent vasodilators cannot be interpreted only as evidence for decreased NOS activity. (J. Clin. Invest. 1995. 96:631-638.) Key words: norepinephrine-induced acute renal failure $\bullet$ acetylcholine $\cdot$ prostacyclin $\cdot$ L-NAME $\cdot$ aminoguanidine

\section{Introduction}

Recent studies have demonstrated that ischemic or hypoxic injury to vital organs, including brain, lung, heart, and kidney, not only damages parenchymal cells but also affects the function and reactivity of the vasculature serving these organs (1-7). A general feature of the altered vasoreactivity is an augmented sensitivity to vasoconstrictor stimuli which, in turn, increases the vulnerability of the affected organ to additional ischemic attacks. Prinzmetal's and postinfarction angina, accelerating transient cerebral ischemic attacks, vasoconstriction after limb ischemia, and prolonged acute renal failure, at least in part, are thought to be clinical examples of postischemic hypersensitivity of the resistance vasculature (1-8).

Since the discovery of endothelium-derived relaxing factor $(E D R F)^{1}(9)$ and identification of its active component as nitric oxide (NO) (10), a commonly held explanation for the augmented constrictor agonist sensitivity of the vasculature has been an ischemia-induced attenuation of EDRF (or NO) activity. NO has been shown to be constitutively generated and released by vascular endothelial cells to continuously modulate the tone and sensitivity of the underlying smooth muscle cells (11). In the absence of NO modulation, there is a leftward shift in agonist sensitivity and increased tone in resistance vessels (12). The hypothesis that ischemia-related changes in vascular sensitivity are due largely to reduced EDRF activity has been supported by the observation that there is a blunted response to endothelium-dependent vasodilators such as acetylcholine ( $\mathrm{ACh})$, bradykinin (BK), serotonin, and histamine in the coronary, cerebral, pulmonary, and renal vasculature after temporary cessation or reduction in organ blood flow or oxygen tension (1-7).

1. Abbreviations used in this paper: $\mathrm{ACh}$, acetylcholine; $\mathrm{AG}$, aminoguanidine; ARF, acute renal failure; BK, bradykinin; $C_{\text {In }}$, inulin clearance; cNOS, constitutive NOS; EDRF, endothelium-derived relaxing factor; iNOS, inducible NOS; L-NAME, nitro-L-arginine methylester; MAP, mean arterial pressure; NO, nitric oxide; NOS, nitric oxide synthase; $\mathrm{PGI}_{2}$, prostacyclin; RBF, renal blood flow; SNP, sodium nitroprusside. 
The kidney arterial vasculature in models of postischemic acute renal failure (ARF) shows constrictor hypersensitivity to renal nerve stimulation (6) and to angiotensin II (13). These models also show attenuated vasorelaxation to ACh and BK (6, 7). Thus, the vasculature of the kidney in postischemic ARF demonstrates functional aberrations similar to those described for other vital organs after ischemia or hypoxia.

The original purpose of this study was to confirm the role of reduced NO activity in the abnormal vascular reactivity of postischemic ARF by examining the effects of the NO synthase (NOS) inhibitor, nitro-L-arginine methylester (L-NAME). It was hypothesized that in the presence of reduced NOS activity L-NAME would not produce the renal vasoconstrictor effect observed in the normal kidney (14). However, it was found that L-NAME infusion, in fact, resulted in a significantly greater renal vasoconstrictor response than in normal or sham-ARF kidneys. The marked vasoconstriction to L-NAME was observed despite the absence of vasodilation to ACh and BK, and a normal vasorelaxation to non-endothelium-dependent prostacyclin $\left(\mathrm{PGI}_{2}\right)$. These findings, in addition to a blunted vasodilatory response to the NO-donor sodium nitroprusside (SNP) and the immunologic detection of constitutive (c) NOS in the endothelium, suggested that NOS activity in the postischemic renal vasculature, rather than being reduced, was present and probably maximal and could not be stimulated further by endothelium-dependent dilators. Furthermore, the absence of a vasoconstrictor response to the inducible NOS (iNOS) inhibitor aminoguanidine (AG) in the ARF vasculature made it likely that the increased NOS was of the constitutive type, probably originating from the endothelium.

\section{Methods}

Renal postischemic injury model. Norepinephrine (NE)-induced ARF has been described previously (15). Briefly, adult Sprague-Dawley rats weighing 250-300 grams underwent right nephrectomy $10 \mathrm{~d}$ before disease induction. After pentobarbital anesthesia, the left renal pedicle was exposed and $\mathrm{NE}$ was delivered at $0.6 \mu \mathrm{g} / \mathrm{kg} \cdot \mathrm{min}^{-1}$ for $90 \mathrm{~min}$ into the renal artery. The rats were returned to metabolic cages receiving water ad libitum and a low-potassium diet (ICN Pharmaceuticals, Cleveland, $\mathrm{OH}$ ) for $72 \mathrm{~h}$ and a standard rat chow diet (Wayne Feed, Longmont, CO) thereafter. Peak azotemia occurred between 24 and $48 \mathrm{~h}$ after NE infusion. Renal blood flow, which was $10-15 \%$ of control during ARF induction, returned to $70 \%$ of control levels by $24 \mathrm{~h}$ and to near control levels by 1 wk $(15)$. Inulin clearance $\left(C_{\text {In }}\right)$ fell to zero after NE infusion and slowly rose to $30 \%$ of control at 1 wk (15). Sham-ARF rats were similarly treated, but $0.9 \%$ saline was substituted for NE infusion into the renal artery.

The techniques of measuring renal vascular reactivity have been described previously (15) and were carried out as follows. At 1 wk, a catheter was placed in the right jugular vein and the left kidney was exposed through a flank incision under pentobarbital anesthesia. The renal artery was dissected bluntly from the renal vein, and a catheter was placed in the ureter. Mean arterial pressure (MAP) was measured through a femoral artery catheter, which was connected to an electronic transducer (P23Db; Stratham Instruments, Oxnard, CA) and a directwriting recorder (model 7702B; Hewlett-Packard Co., Palo Alto, CA). Renal blood flow (RBF) was measured with a miniature electromagnetic flow probe (Carolina Medical Electronics, Inc., King, NC) which was placed around the renal artery and attached to an oscilloscope and digital recorder. The flow probe size was chosen that circumferentially fit closely about the arterial wall by low-power microscopic observation but did not alter urine flow rate. Calibration was conducted according to the method of Arendshorst et al. (16). Ringer's lactate, containing an amount of inulin sufficient to give plasma concentrations of 50-100 $\mathrm{mg} / \mathrm{dl}$, was infused at $2 \mathrm{ml} / \mathrm{h}$ through the jugular venous catheter. After $1 \mathrm{~h}$ of equilibration, blood and urine samples were collected for $\mathrm{C}_{\mathrm{In}}$ measurements. Thereafter, the following protocols were carried out. For each experiment $n=5$ for both NE- and sham-ARF rats.

Responses to EDRF-dependent and EDRF-independent vasodilators. A micropipette ( $35 \mu \mathrm{m}$ OD) was positioned in the renal artery just proximal to the blood flow probe. Baseline RBF measurement was made during $0.9 \%$ saline infusion through the pipette $(2 \mu \mathrm{l} / \mathrm{min})$. The infusion was then changed to one containing $\mathrm{ACh}\left(512 \times 10^{-4} \mu \mathrm{g} / \mathrm{kg} /\right.$ $\min )$, BK $(0.5 \mu \mathrm{g} / \mathrm{kg} / \mathrm{min})$, or $\mathrm{PGI}_{2}\left(3 \times 10^{-4} \mu \mathrm{g} / \mathrm{kg} / \mathrm{min}\right)$. The intrarenal infusion doses were the maximum that produced negligible effects on systemic hemodynamics. After 20 min of infusion RBF measurements were repeated. Vasodilator infusion was then discontinued, and RBF was observed until it had again returned to a stable baseline.

Responses to $L-N A M E$ and $A G$. In separate anesthetized NE- and sham-ARF rats, after baseline RBF measurements were determined, LNAME ( $10 \mathrm{mg} / \mathrm{kg}$ ) was given intravenously over $5 \mathrm{~min} .30 \mathrm{~min}$ later, at the time of stable peak response, RBF was again recorded. The response to L-NAME at the same dose and by the same route was determined a second time after intrarenal infusion of L-arginine $(5 \mathrm{mg} /$ $\mathrm{kg} / \mathrm{min}$ ) for $15 \mathrm{~min}$ before and for $30 \mathrm{~min}$ after giving L-NAME to determine the NOS specificity of the L-NAME effect. To assure that the renovascular response was not a sympathetic response to hypertension induced by L-NAME, L-NAME was also given directly into the renal artery $(1.1 \mathrm{mg} / \mathrm{kg} / \mathrm{h})$, and $\mathrm{RBF}$ was measured at $30 \mathrm{~min}$. To exclude the possibility that lack of response to $\mathrm{ACh}$ and vasoconstriction to $\mathrm{L}$ NAME were not the result of animal-to-animal variation in vascular reactivity, NE-ARF rats were first given ACh $\left(512 \times 10^{-4} \mu \mathrm{g} / \mathrm{kg} /\right.$ min) intrarenally. After $20 \mathrm{~min}, \mathrm{RBF}$ was measured. ACh was then discontinued, and L-NAME (10 $\mathrm{mg} / \mathrm{kg}$ ) was given intravenously to these same rats and RBF was recorded 30 min later.

In separate NE-ARF rats, after determining baseline RBF, the iNOSselective inhibitor AG (17-19) was infused intravenously at $100 \mu \mathrm{M} /$ kg over $5 \mathrm{~min}$. RBF was observed over $60 \mathrm{~min}$. Thereafter, L-NAME $(10 \mathrm{mg} / \mathrm{kg}$ ) was given intravenously and RBF was recorded at $30 \mathrm{~min}$. To determine the iNOS blocking effectiveness of this dose of AG in vivo, normal Sprague-Dawley rats were given AG or vehicle as a bolus intravenous infusion $30 \mathrm{~min}$ before high dose $(10 \mathrm{mg} / \mathrm{kg})$ intravenous infusion of Escherichia coli lipopolysaccharide (LPS) antigen 0127:B8 (Difco Laboratories Inc., Detroit, MI). MAP was monitored over 180 min. In vehicle-treated rats $(n=4)$, MAP fell from a baseline value of $99 \pm 5$ to $73 \pm 7 \mathrm{mmHg}$ at $60 \mathrm{~min}, 68 \pm 8 \mathrm{mmHg}$ at $120 \mathrm{~min}$, and $71 \pm 6$ $\mathrm{mmHg}$ at $180 \mathrm{~min}$ after LPS (all $P<0.01$ compared with baseline). In AG-treated rats $(n=4)$, MAP was $100 \pm 6 \mathrm{mmHg}$ at baseline, $96 \pm 7$ $\mathrm{mmHg}$ at $60 \mathrm{~min}, 99 \pm 6 \mathrm{mmHg}$ at $120 \mathrm{~min}$, and $110 \pm 10 \mathrm{mmHg}$ at 180 min after LPS. None of the MAP values was significantly changed from baseline. The absolute and percent declines in MAP were both significantly greater in the vehicle-treated group $(P<0.01)$. Thus, AG was likely an effective pharmacologic blocker of iNOS at this dose in rats.

Response to SNP. After recording baseline RBF in NE- and shamARF rats, SNP ( $3 \mu \mathrm{g} / \mathrm{kg} / \mathrm{min}$ ) was infused intrarenally through a micropipette as described above. After $10 \mathrm{~min}$ of infusion, RBF was recorded, SNP infusion was discontinued, and RBF was allowed to return to a stable baseline.

Immunologic determinations of endothelial NOS. The immunologic techniques for detecting cNOS were adapted from those of Pollock et al. (20). Kidney tissue was obtained from the upper and lower poles and two midportions of the cortex of sham- and NE-ARF rats that showed vasodilation and lack of vasodilation, respectively, to $\mathrm{ACh}$. For immunohistochemistry, tissue samples no larger than $0.5 \times 1.0 \times 1.0$ $\mathrm{cm}$ were quickly embedded in OCT compound and frozen in ice-cold 2 -methylbutane. Tissue sections of $5 \mu \mathrm{m}$ were cut onto poly-L-lysinecoated slides. The slides were air dried and then fixed in acetone. Nonspecific binding was blocked by immersing the slides in $10 \%$ horse serum in PBS for $40 \mathrm{~min}$; then endogenous biotin was blocked with Biotin Blocking Kit (Vector Labs, Inc., Burlingame, CA) as per manu- 


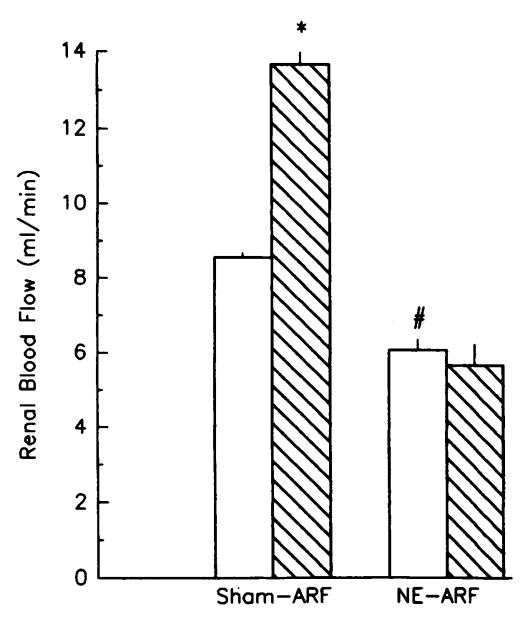

Figure 1. RBF responses to intrarenal infusion of ACh (hatched bars) in sham- and NE-ARF rats. There was a significant increase $\left({ }^{*} P<0.001\right)$ in RBF from baseline (open bars) in sham-ARF. While baseline RBF was lower in NE-ARF kidneys (\# $P<0.01$ ), there was no significant change in $\mathrm{RBF}$ to $\mathrm{ACh}$ infusion. facturer's specifications. Sections were then incubated overnight with anti-NOS monoclonal antibody (kindly provided by Dr. Jennifer Pollock, Abbott Laboratories, North Chicago, IL) at a 1:500 dilution in PBS with 1\% BSA. For negative control, IgG antibody (Zymed Laboratories Inc., South San Francisco, CA) was used at the same dilution. After washing in PBS $(3 \times 10 \mathrm{~min})$, endogenous peroxidase activity was suppressed by a 15 -min incubation in $6 \% \mathrm{H}_{2} \mathrm{O}_{2}$. Then sections were incubated with biotinylated horse anti-mouse IgG 1:40 for $30 \mathrm{~min}$, followed by freshly prepared streptavidin-biotin-horse radish peroxidase (Vector Labs, Inc.) 1:200 for $40 \mathrm{~min}$. After a final PBS wash, the slides were developed with $0.5 \%$ diaminobenzidine and $3 \% \mathrm{H}_{2} \mathrm{O}_{2}$. Hematoxylin was used for counterstaining the slides which were then rinsed in tap water, dehydrated, and mounted. The slides were examined with a Nikon Optiphot microscope. For immunofluorescence, tissue sections from sham- and NE-induced ARF rat kidneys showing response and no response to ACh, respectively, were obtained and quickly frozen as described previously. The technique for tissue preparation was similar to that outlined for immunohistochemistry up to the overnight incubation with the primary antibody and the control. Then, after a PBS wash, the slides were incubated with FITC/anti-mouse IgG conjugate 1:40 (Sigma Immunochemicals, St. Louis, MO) for $1 \mathrm{~h}$. After a final wash, the slide were mounted and then examined with a Nikon Optiphot microscope. The percentages of arcuate and interlobular arteries and arterioles showing positive staining and fluorescence were counted blindly for each slide. A semiquantitative estimate of completeness and intensity of endothelial staining or fluorescence was made.

Analytical procedures. Plasma and urine inulin were measured with an autoanalyzer (Technicon Instruments Corp., Tarrytown, NY). Data are expressed as means $\pm S D$. $C_{\mathrm{In}}$ and renovascular resistance were calculated by standard equations. One-way ANOVA for repeated measures with Bonferroni correction was used to compare time-related functional parameters within each group (21). Comparisons of parameters between groups were made by ANOVA for multiple groups (22). Significance was taken as $P<0.05$.

\section{Results}

Inulin clearances. The levels of $\mathrm{C}_{\mathrm{In}}$ at $1 \mathrm{wk}$ for $\mathrm{NE}-$ and shamARF rats, respectively, were similar for animals used in all separate hemodynamic measurement protocols. The mean value for sham-ARF kidneys was $1.04 \pm 0.10$ and that for NE-ARF kidneys was $0.37 \pm 0.05$, different at $P<0.001$.

Responses to EDRF-dependent and EDRF-independent vasodilators. In both sham- and NE-ARF rats given intrarenal $\mathrm{ACh}$, there were slight but significant declines in MAP from $109 \pm 6$ to $101 \pm 4 \mathrm{mmHg}$ and from $112 \pm 7$ to $100 \pm 5 \mathrm{mmHg}$, respectively $(P<0.05)$. As illustrated in Fig. 1, RBF increased

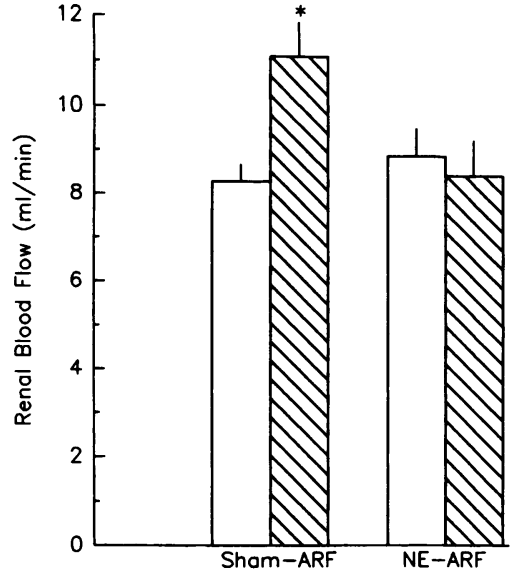

Figure 2. RBF responses to intrarenal BK (hatched bars) in shamand NE-ARF rats. There was a significant increase $(* P<0.001)$ in $\mathrm{RBF}$ from baseline (open bars) in sham-ARF kidneys, but none in NEARF kidneys.

from $8.6 \pm 0.1$ to $13.7 \pm 1.1 \mathrm{ml} / \mathrm{min}(P<0.001)$ in sham-ARF kidneys. In NE-ARF rats, baseline RBF was lower than in shamARF animals $(6.1 \pm 0.3$ vs. $8.6 \pm 0.1 \mathrm{ml} / \mathrm{min}, P<0.01)$. With ACh infusion, $\mathrm{RBF}$ was unchanged at $5.7 \pm 0.6 \mathrm{ml} / \mathrm{min}$ in NEARF kidneys.

The effects of BK on RBF were similar to those with ACh as shown in Fig. 2. Baseline MAPs were similar at $116 \pm 6$ and $114 \pm 8 \mathrm{mmHg}$ in NE- and sham-ARF kidneys, respectively. There were no significant reductions in MAP during intrarenal BK infusion. In sham-ARF rats, RBF increased from $8.3 \pm 0.4$ to $11.1 \pm 0.8 \mathrm{ml} / \mathrm{min}(P<0.001)$, whereas in NE-ARF animals RBF was unchanged $(8.8 \pm 0.6$ vs. $8.4 \pm 0.8 \mathrm{ml} / \mathrm{min})$.

The RBF responses to EDRF-independent $\mathrm{PGI}_{2}$ are shown in Fig. 3. Baseline MAPs were similar at $114 \pm 5$ and $116 \pm 7$ $\mathrm{mmHg}$, respectively, in sham- and NE-ARF rats. There was no significant change in MAP with intrarenal $\mathrm{PGI}_{2}$ in either group. RBF increased from $8.6 \pm 0.5$ to $12.0 \pm 0.9 \mathrm{ml} / \mathrm{min}(P<0.001)$ in sham-ARF kidneys and from $8.4 \pm 0.7$ to $11.2 \pm 0.9 \mathrm{ml} / \mathrm{min}$ $(P<0.001)$ in NE-ARF kidneys. The absolute and relative increases in $\mathrm{RBF}$ in response to $\mathrm{PGI}_{2}$ were similar in the two groups.

Responses to L-NAME and AG. In both sham- and NE-ARF rats there were significant and similar increases in MAP after intravenous L-NAME infusion from $110 \pm 6$ to $135 \pm 13 \mathrm{mmHg}$ and from $112 \pm 6$ to $140 \pm 6 \mathrm{mmHg}$, respectively (both $P$ $<0.001$ ). In the former group RBF fell from $8.8 \pm 0.4$ to $5.5 \pm 0.9 \mathrm{ml} / \mathrm{min}(P<0.001)$, and in the latter group RBF

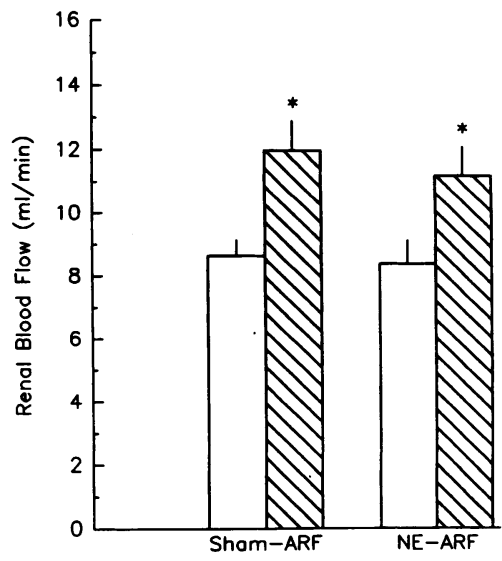

Figure 3. RBF responses to intrarenal infusion of $\mathrm{PGI}_{2}$ (hatched bars) in sham- and NE-ARF rats. There were significant and similar increases $(*$ $P<0.001$ ) in RBF from baseline (open bars) in both groups of rats. 


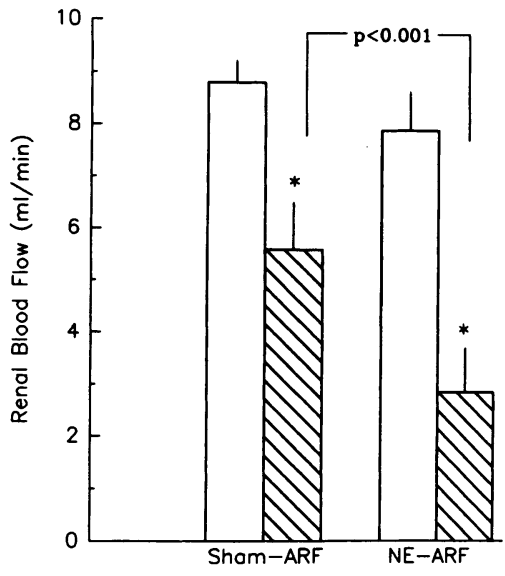

Figure 4. Changes from baseline (open bars) in $\mathrm{RBF}$ after intravenous administration of $\mathrm{L}$ NAME (hatched bars) in sham- and NE-ARF rats. There were significant declines in RBF in both groups $(* P$ $<0.001)$; however, the fall was significantly greater in NE- than sham-ARF kidneys.

declined from $7.9 \pm 0.7$ to $2.8 \pm 0.8 \mathrm{ml} / \mathrm{min}(P<0.001)$ as illustrated in Fig. 4. The baseline RBF were similar for the two groups. However, the absolute $(-5.0 \pm 0.3$ vs. $-2.2 \pm 0.2 \mathrm{ml} /$ min) and fractional ( $-65 \pm 8$ vs. $-37 \pm 5 \%)$ falls in RBF were greater in NE- than sham-ARF kidneys $(P<0.001)$. When $L-$ arginine was infused before and after L-NAME in sham-ARF rats, L-arginine increased RBF slightly, but significantly, from $8.3 \pm 0.5$ to $9.6 \pm 0.7 \mathrm{ml} / \mathrm{min}(P<0.02)$ and MAP was unchanged (112 \pm 7 to $107 \pm 6 \mathrm{mmHg})$. When L-NAME was then given, there was no significant decline in RBF by $30 \mathrm{~min}$ $(9.0 \pm 0.5$ vs. $9.6 \pm 0.7 \mathrm{ml} / \mathrm{min})$, but there was a small increase in MAP $(107 \pm 6$ to $118 \pm 8 \mathrm{mmHg}, P<0.05)$. In NE-ARF rats, there also was no renal vasoconstrictor response to $L$ NAME after L-arginine. Before $\mathrm{L}$-arginine, MAP and RBF were $109 \pm 2 \mathrm{mmHg}$ and $8.0 \pm 0.4 \mathrm{ml} / \mathrm{min}$, respectively. During Larginine, MAP was $110 \pm 1 \mathrm{mmHg}$ and RBF was $7.9 \pm 0.4 \mathrm{ml} /$ min. For $30 \mathrm{~min}$ after L-NAME, MAP increased by only $15 \pm 4$ $\mathrm{mmHg}(P<0.01$ compared with L-NAME alone $)$ and RBF did not change. The minimum recorded value at $30 \mathrm{~min}$ was $7.8 \pm 0.7 \mathrm{ml} / \mathrm{min}$, not different from the before L-NAME value.

Other NE-ARF rats were first infused intrarenally with ACh and then given intravenous L-NAME. Baseline RBF was $6.69 \pm 0.30 \mathrm{ml} / \mathrm{min}$ at a MAP of $110 \pm 5 \mathrm{mmHg}$. With ACh infusion, MAP fell slightly to $105 \pm 4 \mathrm{mmHg}(P<0.05)$ and RBF remained unchanged at $5.96 \pm 0.61 \mathrm{ml} / \mathrm{min}$. When $\mathrm{L}$ NAME was then given intravenously, MAP increased to $135 \pm 8$ $\mathrm{mmHg}(P<0.001)$ and $\mathrm{RBF}$ fell to $3.7 \pm 0.2 \mathrm{ml} / \mathrm{min}(P$ $<0.01)$. This MAP and RBF response sequence is demonstrated in Fig. 5.

The results with direct intrarenal infusion of L-NAME were as follows. In sham-ARF rats with mean baseline MAP and $\mathrm{RBF}$ of $105 \pm 3 \mathrm{mmHg}$ and $8.4 \pm 0.3 \mathrm{ml} / \mathrm{min}$, respectively, intrarenal L-NAME did not increase MAP $(107 \pm 4 \mathrm{mmHg})$ but did decrease RBF by $35 \pm 8 \%$ to $5.4 \pm 0.2 \mathrm{ml} / \mathrm{min}(P<0.01)$. In NE-ARF animals, intrarenal L-NAME also did not change MAP $(120 \pm 8$ vs. $116 \pm 7 \mathrm{mmHg})$, but $\mathrm{RBF}$ fell from $7.6 \pm 1.1$ to $2.7 \pm 1.2 \mathrm{ml} / \mathrm{min}(P<0.001)$. The decreases in RBF with intrarenal L-NAME were quantitatively similar to those with intravenous L-NAME in both sham- and NE-ARF rats despite the lack of changes in MAP. Likewise, the greater decline in RBF to LNAME infusion in NE-ARF compared with sham-ARF rats was similarly observed with intrarenal L-NAME $(P<0.001)$.

The effects of the iNOS inhibitor AG on MAP and RBF, compared with L-NAME, are shown in Fig. 6 for NE-ARF rats.

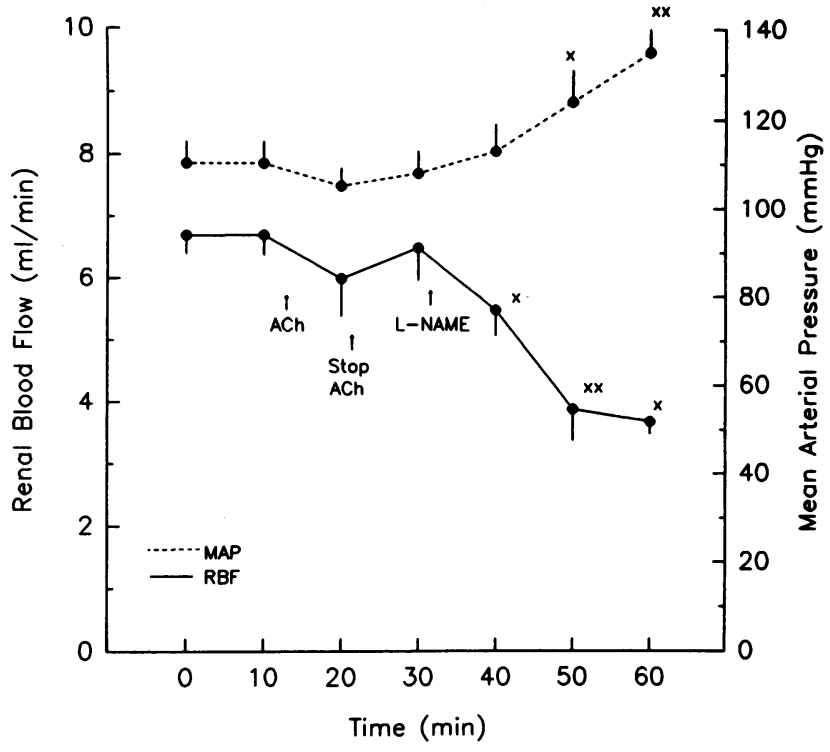

Figure 5. Effects of sequential intrarenal infusion of $\mathrm{ACh}$ followed by intravenous administration of L-NAME on MAP and RBF in NE-ARF rats. ACh did not have significant effects on either parameter. However, L-NAME caused a significant increase in MAP and a significant decrease in RBF. ${ }^{x}$ Different from baseline at $P>0.01$. ${ }^{\mathrm{x}}$ Different from baseline and preceding value at $P<0.01$.

Baseline RBF was $7.7 \pm 0.5 \mathrm{ml} / \mathrm{min}$ at an MAP of $112 \pm 5$ $\mathrm{mmHg}$. RBF and MAP were not changed in $60 \mathrm{~min}$ of AG infusion $(7.8 \pm 0.8 \mathrm{ml} / \mathrm{min}$ and $112 \pm 6 \mathrm{mmHg})$. L-NAME was then given and after $30 \mathrm{~min} R B F$ had fallen to $2.3 \pm 0.2 \mathrm{ml} / \mathrm{min}$ $(P<0.001)$ and MAP had increased to $142 \pm 4 \mathrm{mmHg}(P$ $<0.001)$.

Response to SNP. The changes in MAP and RBF when SNP was infused intrarenally in sham- and NE-ARF rats are

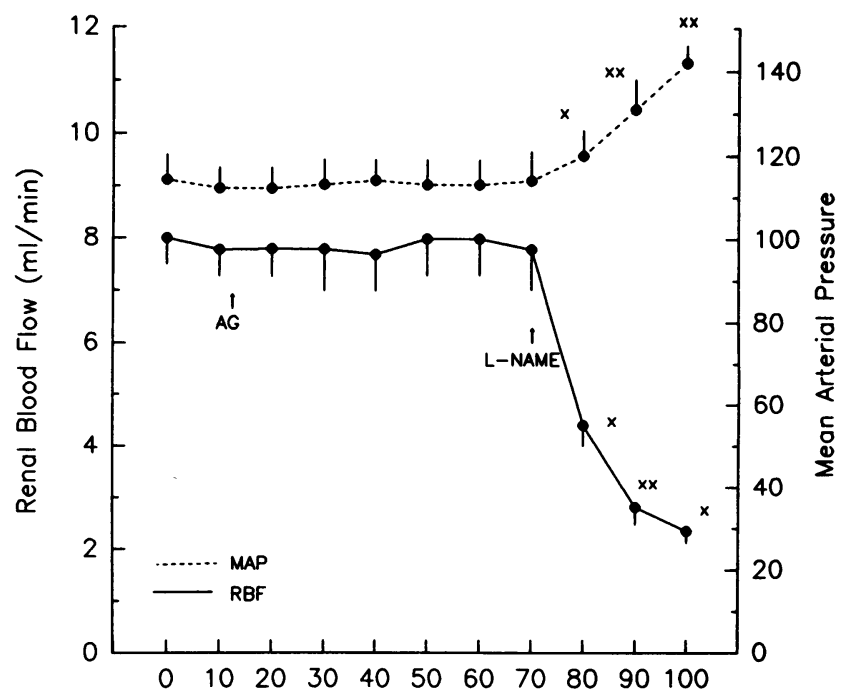

Figure 6. Effects of sequential intravenous administration of AG and L-NAME on MAP and RBF in NE-ARF rats. AG had no effect, but LNAME significantly increased MAP and decreased RBF. ${ }^{\times}$Different from baseline at $P<0.01$. ${ }^{\times}$Different from baseline and preceding value at $P<0.01$. 


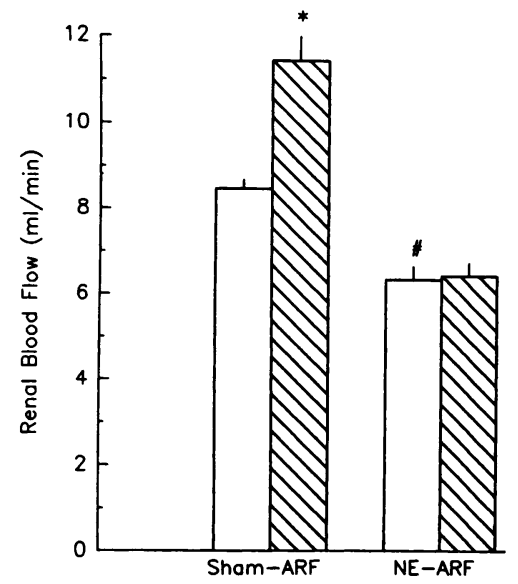

shown in Fig. 7. Baseline MAP fell slightly during SNP infusion from $114 \pm 5$ to $110 \pm 4 \mathrm{mmHg}$ in sham-ARF animals and from $116 \pm 5$ to $110 \pm 5 \mathrm{mmHg}$ in NE-ARF animals (both $P<0.05$ ). $\mathrm{RBF}$ increased from $8.4 \pm 0.2$ to $11.4 \pm 1.4 \mathrm{ml} / \mathrm{min}$ in the former group $(P<0.001)$. Baseline RBF in NE-ARF kidneys was lower than that in sham-ARF kidneys $(6.4 \pm 0.3 \mathrm{vs} .8 .8 \pm 0.2 \mathrm{ml} /$ min, $P<0.01)$. With SNP infusion RBF was unchanged at $6.5 \pm 0.3 \mathrm{ml} / \mathrm{min}$ in NE-ARF kidneys. The absolute $(2.6 \pm 0.2$ vs. $0.1 \pm 0.1 \mathrm{ml} / \mathrm{min})$ and fractional $(30 \pm 7$ vs. $2 \pm 1 \%)$ increases in RBF, respectively, were greater in sham- than NE-ARF kidneys (both $P<0.001$ ).

Immunohistochemistry/fluorescence detection of cNOS. Frozen sections prepared from four cortical tissue slices of five sham-ARF and five NE-ARF kidneys were processed for both cNOS monoclonal antibody and IgG control detection by immunohistochemical and immunofluorescence techniques (minimum of five sections each). The slides were examined for staining or fluorescence of arcuate and interlobular arteries and arterioles. An average of 10 vessels was identified on each slide which was similar in sham- and NE-ARF kidneys. IgG control sections from all kidneys were negative for endothelium-specific staining or fluorescence. Monoclonal antibody to cNOS was detected by both immunohistochemistry and immunofluorescence in the endothelium of $90 \%$ of the vessels of interest in the tissue sections of both sham- and NE-ARF. While not quantifiable by rigid criteria, the intensity of staining (fluorescence) appeared to be greater in the slides from NE-ARF kidneys. Control IgG and cNOS monoclonal antibody staining and fluorescence are illustrated in Figs. 8 and 9.

\section{Discussion}

Decreased response to endothelium-dependent vasodilators, but intact response to endothelium-independent vasodilators, generally has been considered to be evidence in support of reduced
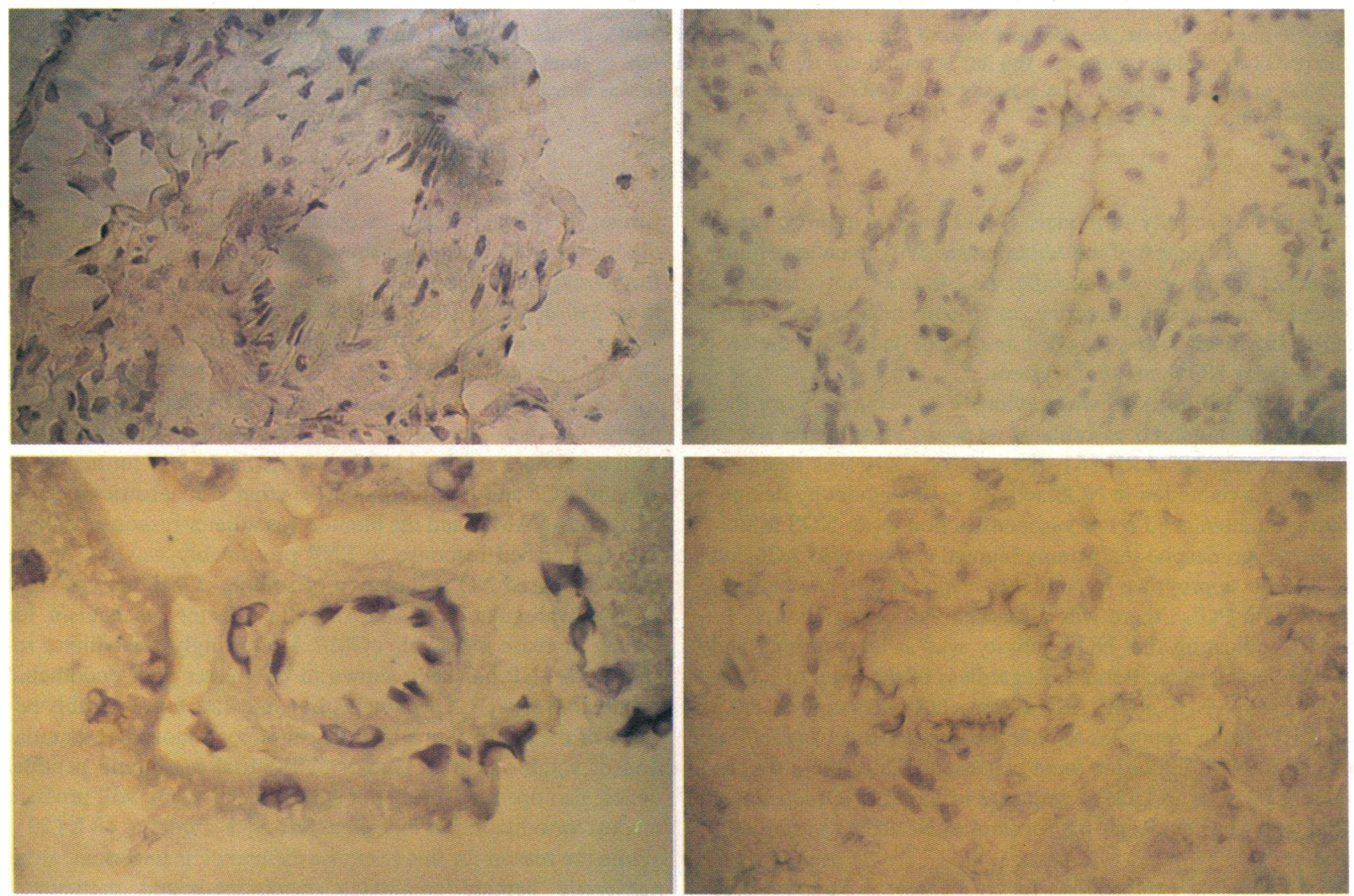

Figure 8. Immunohistochemistry demonstration of typical staining with mouse control IgG antibody or anti-NOS monoclonal antibody in rat renal distal interlobular arteries. Top left is control and top right is anti-NOS in sham-ARF kidney. Bottom left is control and bottom right is anti-NOS in NE-ARF kidney. The endothelial staining with anti-NOS is similar in the sham- and NE-ARF kidneys. 

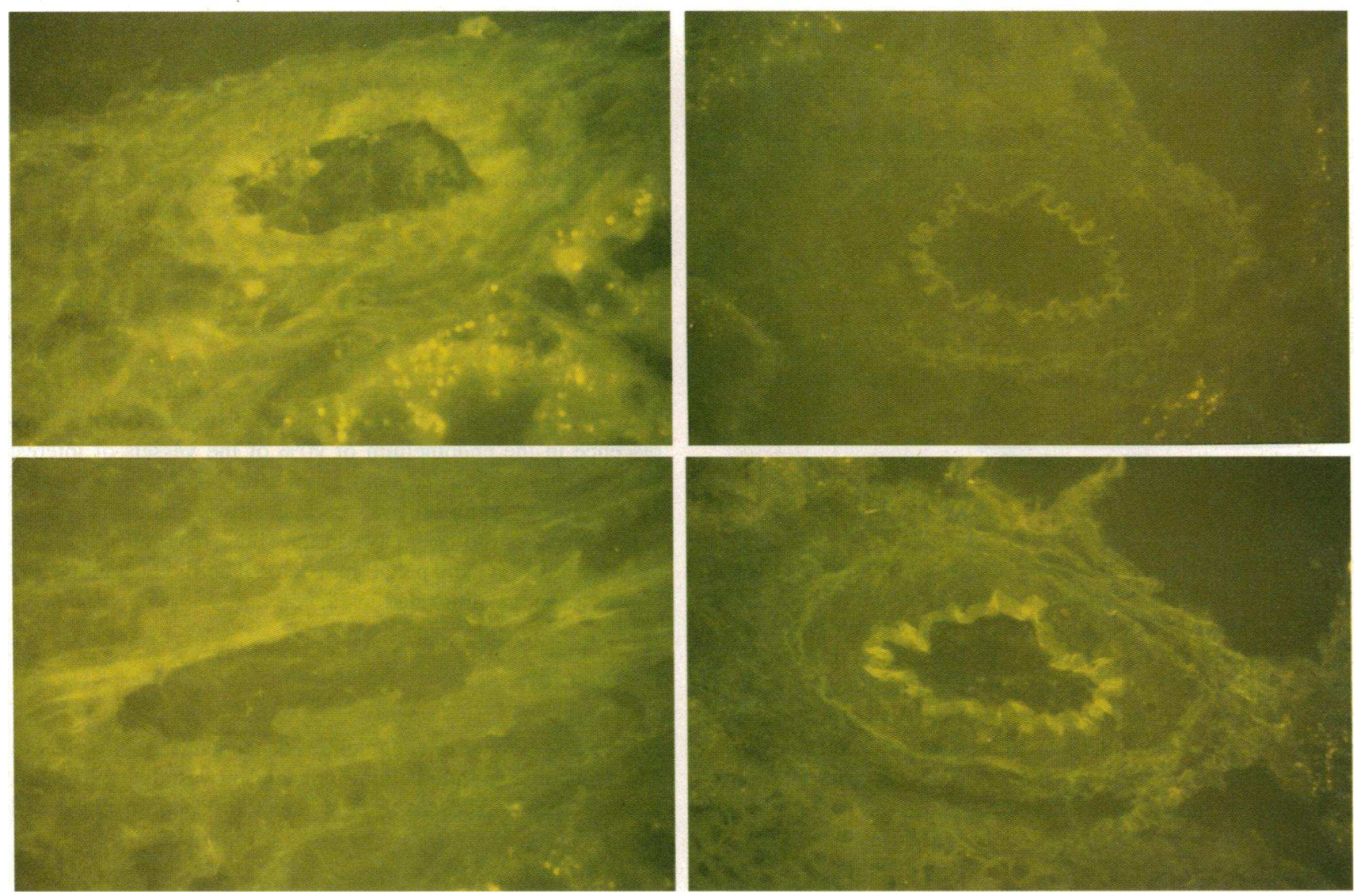

Figure 9. Immunofluorescence demonstration of representative sections of distal interlobular arteries incubated with mouse control IgG antibody or anti-NOS monoclonal antibody. Top left is control and top right is anti-NOS in sham-ARF kidney. Bottom left is control and bottom right is anti-NOS in NE-ARF kidney. The intensity of anti-NOS immunofluorescence was at least similar, if not greater, in the endothelium of NE-ARF compared with sham-ARF kidneys.

EDRF or NO activity in models of ischemic or hypoxic organ injury (1-7). The test of vasodilator sensitivity to endotheliumdependent agents is not specific, i.e., it does not identify a particular endothelial, paracrine, or smooth muscle cell abnormality. However, it does suggest an overall defect in the effective vascular NO generation system.

Since it had been shown previously by our laboratory that there was an impaired vasodilator response to $\mathrm{ACh}$ and $\mathrm{BK}$ in 1-wk ischemic NE-ARF (6), the present study was designed to confirm a reduction in NOS activity in this model by demonstrating an attenuated renal vasoconstriction to an NOS inhibitor. However, despite reaffirming blunted responses to ACh and $\mathrm{BK}$ and an appropriate vasodilator response to endotheliumindependent $\mathrm{PGI}_{2}$, there was a marked vasoconstriction to $\mathrm{L}$ NAME, indicating that NOS activity was at least normal and probably greater than normal. Regardless of the level of recovery of baseline RBF in NE-ARF kidneys at $1 \mathrm{wk}$ (range $6.1 \pm 0.3$ to $8.8 \pm 0.6$ ), the responses to endothelium-dependent vasodilators and to NOS inhibition were consistent. Thus, there was an apparent paradox: a lack of response to largely NO-dependent vasodilators but evidence for substantial NOS/NO vasodilator activity.

Two possible explanations for this apparent paradox were considered. First, there was a post-NOS effector defect, or, second, NOS activity and NO generation were already maximal in the basal state in 1-wk NE-ARF and could not be stimulated further by NO-dependent vasodilators. Even though the lack of dilator response to the NO-donor SNP would be consistent, the former explanation was less plausible. The normal vasodilation to cAMP-dependent $\mathrm{PGI}_{2}$ excluded a defect in smooth muscle relaxation potential, and the vigorous vasoconstriction to $L$ NAME indicated that the L-arginine-NOS-NO-cGMP system was functioning. Thus, a post-NOS effector defect seemed unlikely. On the other hand, all of the findings were consistent with basal state maximal NOS activity: the lack of further relaxation to ACh and BK, the increased renal vasoconstrictor sensitivity to L-NAME, and the blunted vasodilator response to SNP. The diminished response to SNP presumably was due to the basal increased NO stimulation of soluble cGMP activity and/ or a downregulation of the receptor-effector mechanism for further increase in soluble cGMP. Interestingly, vasodilator response to SNP has been shown to be greater after endothelial denudation (Raij, L., personal communication), presumably because of the removal of a component of continuous NO stimulation of soluble cGMP. Unlike SNP, atrial natriuretic peptide, which also operates through a cGMP mechanism, will produce modest vasodilation in this same NE-ARF model at $48 \mathrm{~h}$ (23). While the reason for this response difference is uncertain, atrial natriuretic peptide stimulates membrane-bound particulate, rather than soluble, cGMP which may be unaffected by the continual NO stimulation.

The source of the NOS activity was uncertain. While the 
probable site was the endothelium, the finding of perivascular fibrosis and mononuclear cell infiltration about arterial vessels in this ischemic ARF model at 1 wk (15) raised the possibility that the NOS was of the inducible type as the result of cytokine stimulation (24). The lack of an inhibiting (vasoconstrictor) response to AG followed by a strong inhibition by L-NAME in the NE-ARF rats, suggested that the increased NOS was of the constitutive type and probably was localized in the endothelium. Some caution is required, however, in interpreting the results with AG as regards both other potential effects of this agent. In addition to relatively selective inhibition of iNOS, AG also interferes with the formation of advanced glycosylation end products (19) and inhibits diamine oxidase which contributes to inactivation in vivo of histamine (25). The effect of AG on advanced glycosylation end products is not of likely relevance to this study, but it could be argued that an effect to increase histamine activity could obscure a vasoconstrictor response indicative of iNOS inhibition. There are no data to address this issue in ARF. However, it is unlikely that histamine would be an effective vasodilator in the setting of NE-ARF, since its vasorelaxing effect is endothelium mediated, similar to ACh and BK (26). Moreover, the iNOS inhibiting effect of AG in endotoxin-treated vessels was unaffected by histamine blockers in vitro (18). The choice of an in vivo AG dose for these experiments was unexplored previously. The selective iNOS inhibiting effect of AG is largely based on in vitro evidence in rats showing that endotoxin-induced NOS and vasodilation are inhibited, but endothelium-dependent NO vasodilation is not, unless a concentration of AG from 16 to 40 times greater is used (17-19). The dose chosen for the experiments in this study in vivo ( $100 \mu \mathrm{M} / \mathrm{kg}$ ) has been shown previously to have a minimal, but significant, arterial pressure increasing effect in normal rats (17). It was reasoned that if this dose produced a small detectable inhibiting effect on cNOS in vivo and the iNOS sensitivity to AG was 16 to 40 times greater than the dose chosen, it should have been sufficient to block iNOS in vivo similar to the response seen to L-NAME. However, no vasoconstriction was observed. The preliminary experiments in normal Sprague-Dawley rats showing that the same AG dose blocked the vasodilator effect of injected LPS also supports the likelihood that active iNOS in NE-ARF should have been inhibited if it were present. Therefore, it is reasonable to conclude that the NOS activity was not likely to have been of the inducible type and, much more likely, was constitutive NOS.

It had been shown previously that the serum and urinary levels of nitrite/nitrate $\left(\mathrm{NO}_{2} / \mathrm{NO}_{3}\right)$ correlated with endogenous whole animal NO production (27). However, these measurements were not useful in determining kidney generation of NO in ARF. Urinary $\mathrm{NO}_{2} / \mathrm{NO}_{3}$ are primarily derived from glomerular filtration, and filtered $\mathrm{NO}_{2} / \mathrm{NO}_{3}$ are largely reabsorbed by the proximal tubule under physiologic conditions $(27,28)$. In ARF both GFR and proximal tubular function are substantially altered, making urinary measurements of $\mathrm{NO}_{2} / \mathrm{NO}_{3}$ impossible to interpret as estimates of renal NO generation.

Since urinary measurements of NO metabolites were not helpful in attempting to positively demonstrate NOS in NEARF kidneys, the techniques to detect cNOS by monoclonal antibody were incorporated. Endothelial cNOS was at least as readily found in the endothelium of resistance arterial vessels of NE-ARF as in sham-ARF kidneys by histochemistry or fluorescence methods. Immunologic detection did not necessarily confirm enzyme activity. However, it did provide evidence sup- porting the role of endothelial cNOS function in the NE-ARF kidney when coupled with the pharmacologic inhibition data with L-NAME.

Is there a rationale for the apparent marked increase in NOS activity in the setting of 1-wk ischemic ARF? At this point, the argument is hypothetical, but it is not unreasonable to propose that the increased NOS activity is a response to significant vasoconstrictor stimulation in the established ARF kidney. Endogenous vasoconstrictor agonist activity, including endothelin1, thromboxane $A_{2}$, and renin-angiotensin, has been shown to be increased in ischemic ARF models (29-31). These agonists, in addition to endothelin-3, have been shown to induce endothelial NOS activity (32-35). Alternatively, ischemic injury itself may increase smooth muscle cytosolic calcium (36) or increase oxygen radical generation (37) providing potent vasoconstrictor stimulation to which NOS activation is a modulating response. It is of interest that despite evidence of increased constrictor agonist activity in ischemic ARF models, basal RBF is nearly always relatively well preserved in the established phase of disease compared with GFR, suggesting that there may be an opposing vasodilator activity operating simultaneously. The results of this study using L-NAME demonstrated the dramatic vasoconstriction that would occur if NOS activity was actually reduced in 1-wk ischemic ARF. At present it is uncertain what specific mechanism or mediator(s) is responsible for the vasoconstrictor activity unmasked by NOS inhibition.

Regardless of the mechanism of increased, and probably maximal, NOS activity, this finding is actually consistent with other observations in this ischemic ARF model (15). Basal RBF approaches normal. If NOS activity were truly decreased, then basal RBF should be much lower. Despite the nearly normal basal RBF, there is a marked vascular hypersensitivity to additional exogenous constrictor stimuli such as renal nerve stimulation and angiotensin II $(6,13)$. Assumedly, if NOS activity were maximal in the basal state, then further NO generation would not occur to modulate the effect of added constrictor agonist.

It is unknown whether the findings in this study, i.e., that attenuated responses to endothelium-dependent vasodilators may not indicate a reduction in NOS activity, have relevance to reports of postischemic or posthypoxic vascular reactivity in other organs and in essential hypertension as well, where attenuated response to endothelium-dependent vasodilators has also been reported (38). While lack of response to endothelialdependent vasodilators may actually represent a true decrease in NOS activity in heart, lung, or brain, it is of interest to note that in two recent publications postischemic NOS activity was detected. In hypoxic pulmonary vasoconstriction and poststroke vasoreactivity, conditions reported to be associated with decreased ACh vasorelaxation, NOS activity was functionally and immunologically detectable $(39,40)$. Thus, it may be necessary to reassess the interpretation of some studies regarding the meaning of decreased endothelium-dependent vasodilator responses in ischemic and hypoxic injury.

\section{Acknowledgments}

Mary Lee prepared the manuscript. Dr. Jennifer Pollock of Abbott Laboratories advised our laboratory and reviewed endothelial NOS immunofluorescence/immunohistochemistry results. Dr. Ivan McMurty critically reviewed the paper and made helpful comments and suggestions.

This work was supported by National Institutes of Health grant DK35098 and VA research funds. 


\section{References}

1. Mayhan, W. G., S. M. Amundsen, F. M. Faraci, and D. D. Heistad. 1988. Responses of cerebral arteries after ischemia and reperfusion in cats. Am. J. Physiol. 255:H879-H884.

2. Perrella, M. A., E. S. Edell, M. J. Krowka, D. A. Cortese, and J. C. Burnett, Jr. 1992. Endothelium-derived relaxing factor in pulmonary and renal circulations during hypoxia. Am. J. Physiol. 263:R45-R50.

3. Adnot, S., B. Raffestin, S. Eddahibi, P. Braquet, and P. E. Chabrier. 1991. Loss of endothelium-dependent relaxant activity in the pulmonary circulation of rats exposed to chronic hypoxia. J. Clin. Invest. 87:155-162.

4. VanBenthuysen, K. M., I. F. McMurtry, and L. D. Horwitz. 1987. Reperfusion after acute coronary occlusion in dogs impairs endothelium-dependent relaxation to acetylcholine and augments contractile reactivity in vitro. J. Clin. Invest. 79:265-274.

5. Quillen, J. E., F. W. Sellke, L. A. Brooks, and D. G. Harrison. 1990. Ischemia-reperfusion impairs endothelium-dependent relaxation of coronary microvessels but does not affect large arteries. Circulation. 82:586-594.

6. Conger, J. D., J. B. Robinette, and R. W. Schrier. 1988. Smooth muscle calcium and endothelium derived relaxing factor in the abnormal vascular responses of acute renal failure. J. Clin. Invest. 82:532-537.

7. Sternbergh, W. C, R. G. Makhoul, and B. Adelman. 1993. Nitric oxidemediated, endothelium-dependent vasodilation is selectively attenuated in the postischemic extremity. Surgery (St. Louis). 114:960-967.

8. Conger, J. D., M. F. Schultz, F. Miller, and J. B. Robinette. 1994. Responses to hemorrhagic arterial pressure reduction in different ischemic renal failure models. Kidney Int. 46:318-326.

9. Furchgott, R. F., and J. V. Zawadzki. 1980. The obligatory role of endothelial cells in the relaxation of arterial smooth muscle by acetylcholine. Nature (Lond.). 288:373-376.

10. Palmer, R. M. J., A. G. Ferrige, and S. Moncada. 1987. Nitric oxide release accounts for the biological activity of endothelium-derived relaxing factor. Nature (Lond.). 327:524-526.

11. Palmer, R. M. J., and S. Moncada. 1989. A novel citrulline-forming enzyme implicated in the formation of nitric oxide by vascular endothelial cells. Biochem. Biophys. Res. Commun. 158:348-352.

12. Shepherd, J. T., and Z. S. Katusic. 1991. Endothelium-derived vasoactive factors. I. Endothelium-dependent relaxation. Hypertension (Dallas). 18(Suppl. III): III-76-III-85.

13. Conger, J. D., and S. A. Falk. 1993. Abnormal vasoreactivity of isolated arterioles from rats with ischemic acute renal failure. J. Am. Soc. Nephrol. 4:733. (Abstr.)

14. Baylis, C., K. Engles, L. Samsell, and P. Harton. 1993. Renal effects of acute endothelial-derived relaxing factor blockade are not mediated by angiotensin II. Am. J. Physiol. 264:F74-F78.

15. Conger, J. D., J. B. Robinette, and W. S. Hammond. 1991. Differences in vascular reactivity in models of ischemic acute renal failure. Kidney Int 39:1087-1097.

16. Arendshorst, W. J., W. F. Finn, and C. G. Gottschalk. 1979. Autoregulation of renal blood flow in the rat. Circ. Res. 44:344-349.

17. Hasan, K., B. J. Heesen, J. A. Corbett, M. L. McDaniel, K. Chang, W. Allsion, B. H. R. Wolffenbuttel, J. R. Williamson, and R. G. Tilton. 1993. Inhibition of nitric oxide formation in guanidines. Eur. J. Pharmacol. 249:101-106.

18. Griffiths, M. J. D., M. Messent, R. J. MacAllister, and T. W. Evans. 1993. Aminoguanidine selectively inhibits inducible nitric oxide synthase. $B r . J$. Pharmacol. 110:963-968.

19. Tilton, R. G., K. Chang, K. S. Hasan, S. R. Smith, J. M. Petrash, T. P. Misko, W. M. Moore, M. G. Currie, J. A. Corbett, M. L. McDaniel and J. R. Williamson. 1993. Prevention of diabetic vascular dysfunction by guanidines.
Inhibition of nitric oxide synthase versus advanced glycation end-produce formation. Diabetes. 42:221-232.

20. Pollock, J. S., M. Nakane, L. D. K. Buttery, A. Martinez, D. Springall, J. M. Polak, U. Förstermann, and F. Murad. 1993. Characterization and localization of endothelial nitric oxide synthase using specific monoclonal antibodies. Am. J. Physiol. 265:C1379-C1387.

21. Snedecor, G. W., and W. G. Cochran. 1980. Statistical Methods. The Iowa State University, Ames, IA. 221-339.

22. Wallenstein, S., C. L. Zucker, and J. L. Fleiss. 1980. Some statistical methods useful in circulation research. Circ. Res. 47:1-9.

23. Conger, J. D., S. A. Falk, and W. S. Hammond. 1991. Atrial natriuretic peptide and dopamine in established acute renal failure in the rat. Kidney Int. 40:21-28.

24. Gross, S. S., E. A. Jaffe, R. Levi, and R. G. Kilbourne. 1991. Cytokineactivated endothelial cells express an isotype of nitric oxide synthase which is tetrahydrobiopterin dependent, calmodulin-independent, and inhibited by arginine analogs with a rank of order of potency characteristic of activated macrophages. Biochem. Biophys. Res. Commun. 178:823-829.

25. Wolvekamp, M. C., and R. W. deBruin. 1994. Diamine oxidase: and overview of historical, biochemical and functional aspects. Dig. Dis. 12:2-14.

26. Ignarro, L. J. 1989. Biological actions and properties of endotheliumderived nitric oxide formed and released from artery and vein. Circ. Res. 65:121.

27. Shultz, P. J., and L. Raij. 1992. Endogenously synthesized nitric oxide prevents endotoxin-induced glomerular thrombosis. J. Clin. Invest. 90:17181725.

28. Süto, T., G. Losonczy, C. Qiu, K. Engels, and C. Baylis. 1994. Acute changes in urinary $\mathrm{NO}_{2}+\mathrm{NO}_{3}$ excretion $\left(\mathrm{U}_{\mathrm{NO}} \mathrm{V}\right)$ reflect alterations in tubular $\mathrm{NO}_{x}$ reabsorption rather than nitric oxide (NO) production. J. Am. Soc. Nephrol. 5:593A.

29. Shibouta, Y. N. Suzuki, A. Shino, H. Matsumoto, A. Terashita, K. Kondo, and K. Nishikawa. 1990. Pathophysiological role of endothelin in acute renal failure. Life Sci. 46:1611-1619.

30. DiBona, G. F., and L. L. Sawin. 1971. The renin-angiotensin system in cute renal failure in the rat. Lab. Invest. 25:528-532.

31. Hatziantonious, C., and N. Papankolaou. 1986. Renal effects of the inhibitor of thromboxane $A_{2}$-synthetase OKY-046. Experientia (Basel). 42:613-615.

32. Edwards, R. M., M. Pullen, and P. Nambi. 1992. Activation of endothelin $\mathrm{ET}_{\mathrm{B}}$ receptors increases glomerular cGMP via an L-arginine-dependent pathway. Am. J. Physiol. 263:F1020-F1025.

33. Henrich, W. L. 1991. Southwestern internal medicine conference: the endothelium - a key regulator of vascular tone. Am. J. Med. Sci. 302:319-328.

34. Sigmon, D. H., O. A. Carretero, and W. H. Beierwaltes. 1992. Angiotensin dependence of endothelium-mediated renal hemodynamics. Hypertension (Dallas). 20:643-650.

35. Warner, T. D., H. H. Schmidt, and F. Murad. 1992. Interactions of endothelins and EDRF in bovine native endothelial cells: selective effects of endothelin-3. Am. J. Physiol. 262:H1600-H1605.

36. Schrier, R. W., J. D. Conger, and T. J. Burke. 1990. Pathogenetic role of calcium in renal cell injury. Proc. Int. Soc. Nephrol. 11:1-12.

37. Simon, B. C., L. D. Cunningham, and R. A. Cohen. 1990. Oxidized low density lipoproteins cause contraction and inhibit endothelium-dependen relaxation in the pig coronary artery. Am. Soc. Clin. Invest. 86:75-79.

38. Panza, J. A., A. A. Quyyumi, J. E. Bruch, and S. E. Epstein. 1990. Abnormal endothelium-dependent vascular relaxation in patients with essentia hypertension. N. Engl. J. Med. 323:22-27.

39. McMurtry, I., M. Oka, K. Hasunuma, T. Yamaguchi, K. Morris, and D. Rodman. 1992. Role of EDRF in control of normoxic and hypoxic pulmonary vascular tone. High Altitude Medicine.

40. Zhang, Z G., C. Zaloga, J. S. Pollock, and U. Forstermann. 1993. Cerebra endothelial nitric oxide synthase expression after focal cerebral ischemia in rats. Stroke. 24:2016-2021. 